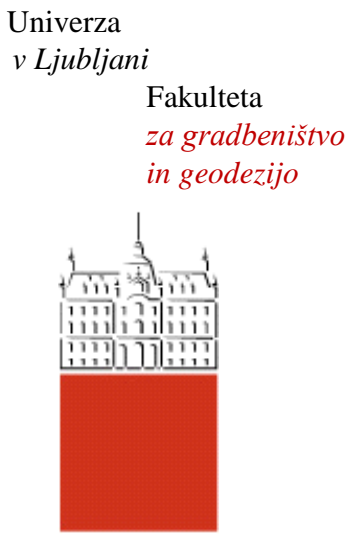

Jamova cesta 2

1000 Ljubljana, Slovenija

http://www3.fgg.uni-lj.si/

\section{DRUGG - Digitalni repozitorij UL FGG http://drugg.fgg.uni-lj.si/}

Ta članek je avtorjeva zadnja recenzirana različica, kot je bila sprejeta po opravljeni recenziji.

Prosimo, da se pri navajanju sklicujte na bibliografske podatke, kot je navedeno:
University
of Ljubljana

Faculty of Civil and Geodetic Engineering

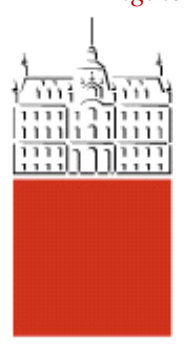

Jamova cesta 2 SI - 1000 Ljubljana, Slovenia http://www3.fgg.uni-lj.si/en/

DRUGG - The Digital Repository http://drugg.fgg.uni-lj.si/

This version of the article is author's manuscript as accepted for publishing after the review process.

When citing, please refer to the publisher's bibliographic information as follows:

Zupan, D., Saje, M. 2008. Convergence properties of materially and geometrically non-linear finite-element spatial beam analysis. Finite elements in analysis and design 45,1: 60-69. DOI: $\underline{10.1016 / j . f i n e l .2008 .07 .013}$ 


\title{
Convergence properties of materially and geometrically non-linear finite-element spatial beam analysis
}

\author{
D. Zupan* and M. Saje \\ University of Ljubljana, Faculty of Civil and Geodetic Engineering, \\ Jamova 2, SI-1115 Ljubljana, Slovenia
}

\begin{abstract}
The way the non-linear constitutive equations in the spatial beam formulations are solved, influences the rate of convergence and the computational cost. Three main different approaches are studied: (i) the direct global approach, where the constitutive equations are taken to be the iterative part of the global governing equations, (ii) the local (or indirect global) approach, where the constitutive equations are solved separately in each step of the global iteration, and (iii) the partly reduced approach, which is the combination of (i) and (ii). The approaches are compared with regard to the number of global iterations and the total number of floating point operations. The direct global approach is found to be the best choice.
\end{abstract}

Key words: non-linear beam theory, non-linear material, reinforced concrete, stress field integration, Newton's iteration, rate of convergence.

\section{Introduction}

In the analysis of structures the non-linearity of the material law often dictates the rate of convergence of the solution procedure. The objective of the present paper is to find out how various approaches for solving the non-linear constitutive equations effect the rate of convergence and the total number of floating point operations in the finite-element analysis of spatial beams and frames. Several approaches for the solution of the discretized equations of the non-linear spatial beams are at hand. The constitutive equations, for example, can be eliminated from the set of the governing equations prior to its solution.

* Corresponding author. E-mail addresss: dejan.zupan@fgg.uni-lj.si 
This reduces the size of the global system of linearized equations that need to be solved in each iteration. When material is non-linear, such a formulation requires solving, in each step of the global iteration and locally in each integration point, the non-linear constitutive equations. This implies that, in each step of the global iteration, an additional number of local iterations need to be executed. This may remarkably increase the computational time. If, in contrast, the constitutive equations in integration points are assumed to be the part of the global governing equations, the size of the overall system of equations is larger. Such an approach may somewhat increase the number of global iterations, but because no additional local iterations are needed, the overall computational cost will probably be lower. Another interesting approach is a mixed approach, where a part of constitutive equations is solved locally and the remaining part is taken as a member of global governing equations.

The influence of three different approaches on the rate of convergence and the overall computational cost is examined through numerical examples. We compare not only the number of iterations, but also the total number of floating point operations, thus assessing also the actual computational cost of the algorithm apart from the actual computer used.

\section{Governing equations of the beam}

The complete set of the beam equations consists of the cross-sectional constitutive equations (1)-(2), the equilibrium equations (3)-(4), the kinematic equations (5)-(6) (see [11], [12], [13])

$$
\begin{aligned}
\mathbf{R}(x) \mathcal{C}_{N}\left(\gamma_{G}(x), \boldsymbol{\kappa}_{G}(x)\right)-\boldsymbol{N}_{g}(x) & =\mathbf{0} \\
\mathbf{R}(x) \mathcal{C}_{M}\left(\gamma_{G}(x), \boldsymbol{\kappa}_{G}(x)\right)-\boldsymbol{M}_{g}(x) & =\mathbf{0} \\
\boldsymbol{N}_{g}^{\prime}(x)+\boldsymbol{n}_{g}(x) & =\mathbf{0} \\
\boldsymbol{M}_{g}^{\prime}(x)+\boldsymbol{m}_{g}(x)-\boldsymbol{N}_{g}(x) \times \mathbf{R}(x)\left(\boldsymbol{\gamma}_{G}(x)-\boldsymbol{c}_{G}(x)\right) & =\mathbf{0} \\
\boldsymbol{r}_{g}^{\prime}(x)-\mathbf{R}(x)\left(\boldsymbol{\gamma}_{G}(x)-\boldsymbol{c}_{G}(x)\right) & =\mathbf{0} \\
\boldsymbol{\vartheta}_{g}^{\prime}(x)-\mathbf{T}^{-T}(x)\left(\boldsymbol{\kappa}_{G}(x)-\boldsymbol{d}_{G}(x)\right) & =\mathbf{0}
\end{aligned}
$$

and the related static boundary conditions:

$$
\begin{array}{r}
\boldsymbol{S}^{0}+\boldsymbol{N}_{g}(0)=\mathbf{0} \\
\boldsymbol{P}^{0}+\boldsymbol{M}_{g}(0)=\mathbf{0} \\
\boldsymbol{S}^{L}-\boldsymbol{N}_{g}(L)=\mathbf{0} \\
\boldsymbol{P}^{L}-\boldsymbol{M}_{g}(L)=\mathbf{0} .
\end{array}
$$

Here, the prime (') denotes the derivative with respect to the arc-length parameter of the line of centroids in the initial configuration, $x$, and " $\times$ " marks 
the cross vector product. The meaning of the notations used in the above equations is described below (see also Fig. 1):

$g$ fixed (inertial) orthonormal basis $\left\{\vec{g}_{1}, \vec{g}_{2}, \vec{g}_{3}\right\}$ spanning the physical space of the beam;

$G$ orthonormal basis $\left\{\vec{G}_{1}, \vec{G}_{2}, \vec{G}_{3}\right\}$ spanning the cross-sectional planes;

$\boldsymbol{N}, \boldsymbol{M}$ stress-resultant force and moment vectors over the cross-section;

$\mathcal{C}_{N}, \mathcal{C}_{M}$ operators describing material of the beam;

$\gamma$ translational strain vector $\left(\gamma_{G 1}\right.$ is the extensional strain, $\gamma_{G 2}, \gamma_{G 3}$ are shear strains);

$\kappa$ rotational strain vector $\left(\kappa_{G 1}\right.$ is the torsional strain, $\kappa_{G 2}, \kappa_{G 3}$ are the curvatures);

$\boldsymbol{r}$ position vector of the line of centroids of the beam;

$\mathbf{R}$ both the rotation matrix from $g$ to $G$ and the coordinate transformation matrix $\left(\boldsymbol{v}_{g}=\mathbf{R} \boldsymbol{v}_{G}\right)$

$\vartheta$ rotational vector whose axis coincides with the axis of rotation and whose length equals the angle of rotation;

$\boldsymbol{\Theta}$ skew-symmetric matrix $\boldsymbol{\Theta}$ composed from its axial vector $\boldsymbol{\vartheta}_{g}=\left[\begin{array}{lll}\vartheta_{g 1} & \vartheta_{g 2} & \vartheta_{g 3}\end{array}\right]^{T}$;

$\mathbf{T}^{T}$ transformation matrix between $\boldsymbol{\kappa}_{G}$ and $\boldsymbol{\vartheta}_{g}^{\prime}$ (i.e., $\mathbf{T}^{T}=\mathbf{I}-\frac{1-\cos \vartheta}{\vartheta^{2}} \boldsymbol{\Theta}+\frac{\vartheta-\sin \vartheta}{\vartheta^{3}} \boldsymbol{\Theta}^{2}$, $\left.\vartheta=\left\|\boldsymbol{\vartheta}_{g}\right\|\right)$

$\boldsymbol{c}, \boldsymbol{d}$ variational constants determined from the known strains, position vectors and rotations in the initial configuration;

$\boldsymbol{n}, \boldsymbol{m}$ external distributed force and moment vectors per unit of the undeformed length of the axis;

$\boldsymbol{S}^{0}, \boldsymbol{S}^{L}$ external point forces at the boundaries $x=0, x=L$;

$\boldsymbol{P}^{0}, \boldsymbol{P}^{L}$ external point moments at the boundaries $x=0, x=L$.

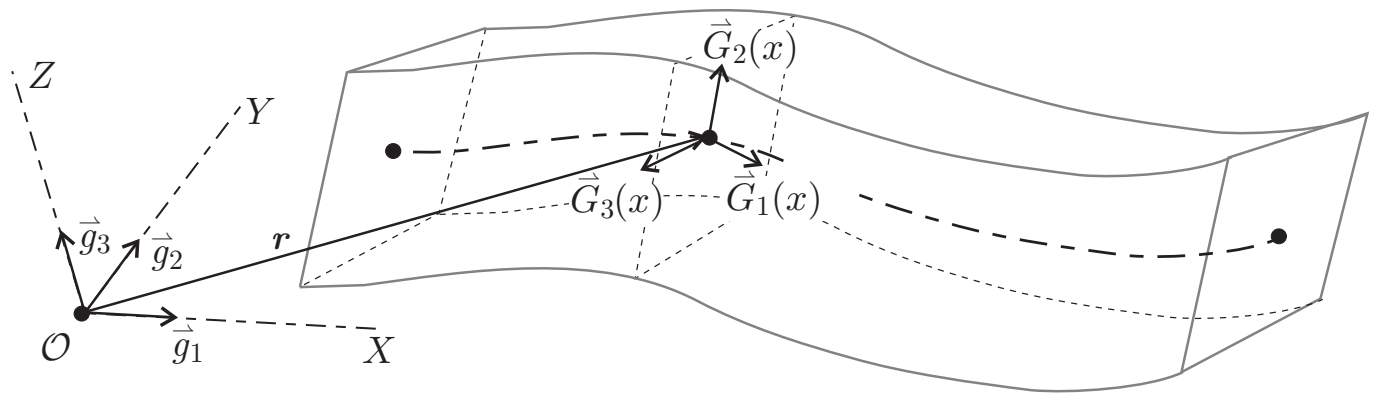

Fig. 1. Model of the three-dimensional beam. 


\section{Reinforced concrete $3 \mathrm{D}$ beams}

Due to its widespread use in practice the reinforced concrete material is found convenient for demonstrating the convergence properties of various approaches. Let us describe the mathematical model of reinforced concrete first.

\subsection{Constitutive law of concrete}

We follow Desayi and Krishnan [3] and Bergan and Holand [1] and employ the uniaxial stress-strain relation for concrete given by the function (see Fig. 2 for its graph):

$$
\sigma(\varepsilon)=\left\{\begin{array}{ll}
0 & \varepsilon \leq \varepsilon_{\mathrm{u}} \\
2 f_{\mathrm{m}}\left|\varepsilon_{1}\right| \frac{\varepsilon}{\varepsilon_{1}^{2}+\varepsilon^{2}} & \varepsilon_{\mathrm{u}}<\varepsilon \leq \varepsilon_{\mathrm{r}} \\
\frac{\sigma_{\mathrm{r}}}{\varepsilon_{\mathrm{r}}-\varepsilon_{\mathrm{m}}}\left(\varepsilon-\varepsilon_{\mathrm{m}}\right) & \varepsilon_{\mathrm{r}}<\varepsilon \leq \varepsilon_{\mathrm{m}} \\
0 & \varepsilon_{\mathrm{m}}<\varepsilon
\end{array} .\right.
$$

Here $f_{\mathrm{m}}$ is ultimate strength of concrete in compression $\left(f_{\mathrm{m}}>0\right) ; \varepsilon_{1}<0$ is the corresponding strain; $\varepsilon_{\mathrm{u}}<0$ is ultimate strain in compression; $\varepsilon_{\mathrm{r}}>0$ is strain at ultimate strength of concrete in tension; and $\varepsilon_{\mathrm{m}}>0$ is ultimate strain in tension. Parameters $f_{\mathrm{m}}, \varepsilon_{1}$, and $\varepsilon_{\mathrm{u}}$ have to be determined in compression tests on concrete cylinders; similarly $\varepsilon_{\mathrm{r}}$ and $\varepsilon_{\mathrm{m}}$ have to be determined by tension tests. Because the tension zone is not essential in modeling concrete, the tension tests are rarely performed in practice and empirically found average values $\varepsilon_{\mathrm{r}}=5.5 \cdot 10^{-5}$ and $\varepsilon_{\mathrm{m}}=7 \cdot 10^{-4}$ are utilized instead [1].

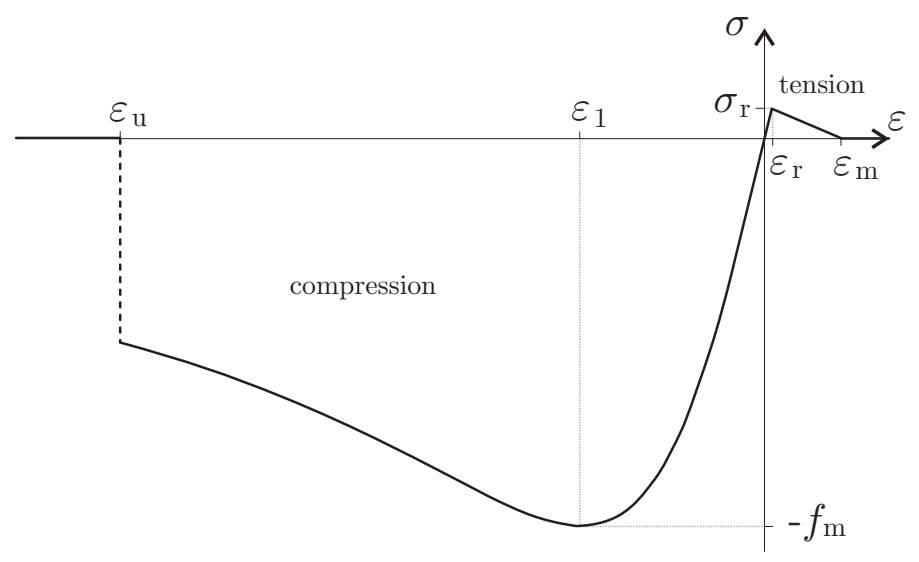

Fig. 2. Constitutive law of concrete. 


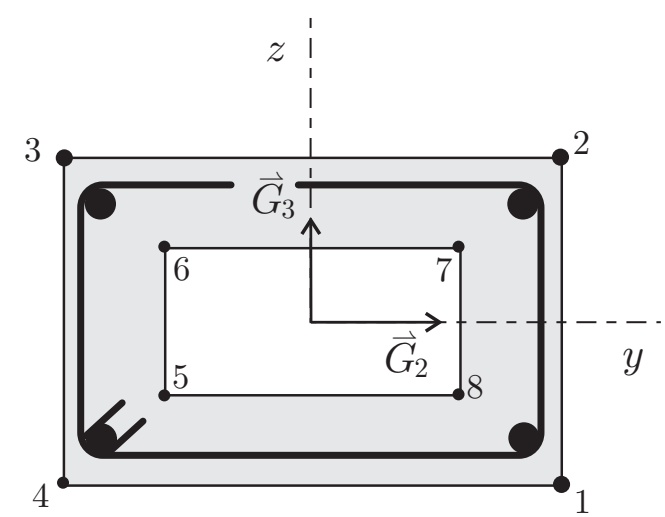

Fig. 3. Model of the cross-section and the local coordinate system $(y, z)$ with the base vectors along the principal axes of inertia of the cross-section.

Note that the function proposed in Eq. (7) is discontinuous at $\varepsilon_{\mathrm{u}}$ and that its first derivative with respect to $\varepsilon$ is discontinuous at $\varepsilon_{\mathrm{u}}, \varepsilon_{\mathrm{r}}$ and $\varepsilon_{\mathrm{m}}$. This property is very inconvenient, because the formation of the tangent stiffness matrix of the beam element is then faced with the integration of a discontinuous function over the cross-section, which requires special measures to be applied.

In spatial beam elements, when used in frame-like structures, we usually assume the Bernoulli hypothesis that a cross-section suffers only rigid translation and rotation during deformation. This implies that the normal strain (axial strain), $\varepsilon$, at the cross-section $(x=$ const.) is linearly distributed over the cross-section:

$$
\varepsilon(y, z)=\gamma_{G 1}-y \kappa_{G 3}+z \kappa_{G 2} .
$$

Here, $y$ and $z$ are the local coordinates at the cross-section defined by base vectors $\vec{G}_{2}$ and $\vec{G}_{3}$ (see also Figure 1 ). The corresponding normal (axial) stress distribution, $\sigma(y, z)$, over the concrete cross-section $x=$ const. is determined from the constitutive law (7). The integration of stresses and their static moments over the cross-sections yields the axial force and the bending moments taken by the concrete part of the cross-section. They are given with respect to the centroid of the cross-section:

$$
\begin{aligned}
N_{G 1 c} & =\iint_{\mathcal{A}} \sigma(\varepsilon(y, z)) d y d z \\
M_{G 2 c} & =\iint_{\mathcal{A}} z \sigma(\varepsilon(y, z)) d y d z \\
M_{G 3 c} & =-\iint_{\mathcal{A}} y \sigma(\varepsilon(y, z)) d y d z .
\end{aligned}
$$

The shear forces and the torsional moment are assumed to depend linearly on 
the resulting shear and torsional strains

$$
\begin{aligned}
& N_{G 2 c}=G A_{2} \gamma_{2} \\
& N_{G 3 c}=G A_{3} \gamma_{3} \\
& M_{G 1 c}=G J_{t} \kappa_{1} .
\end{aligned}
$$

Here, $A_{2}$ and $A_{3}$ are the shear areas; $J_{t}$ is its torsional inertial moment; $G$ is the shear modulus of concrete, here assumed constant and independent on deformation.

\subsection{Constitutive law of reinforcing steel}

The constitutive law of reinforcing steel is taken to be a three-linear law with the same behaviour in tension and compression (Fig. 4):

$$
\sigma_{s}(\varepsilon)= \begin{cases}E_{\mathrm{s}} \varepsilon & |\varepsilon| \leq \varepsilon_{\mathrm{y}} \\ \operatorname{sgn}(\varepsilon)\left(E_{\mathrm{s}} \varepsilon_{\mathrm{y}}+E_{\mathrm{p}}\left(|\varepsilon|-\varepsilon_{\mathrm{y}}\right)\right) & \varepsilon_{\mathrm{y}}<|\varepsilon| \leq \varepsilon_{\mathrm{ym}} . \\ 0 & \varepsilon_{\mathrm{ym}}<|\varepsilon|\end{cases}
$$

$E_{\mathrm{s}}>0$ is elastic modulus of steel; $E_{\mathrm{p}}>0$ is its hardening modulus in the plastic region; $\varepsilon_{\mathrm{y}}>0$ is the strain at yield stress in tension; $\varepsilon_{\mathrm{ym}}>0$ is the ultimate strain (Fig. 4).

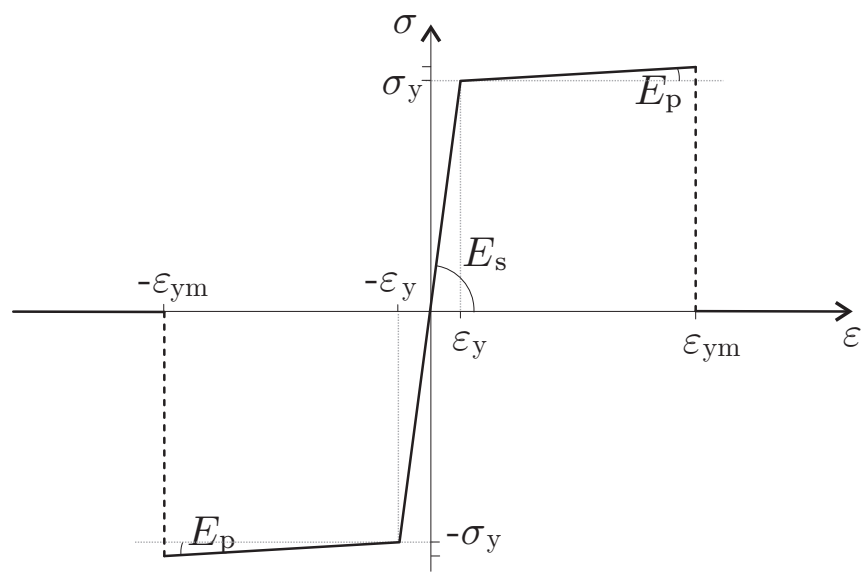

Fig. 4. Constitutive law of reinforcing streel.

Because the area of the reinforcing steel bars is small compared to the area of the concrete cross-section, we assume a constant stress field over each steel bar. As stresses in steel bars can be much larger than the stresses in concrete, we can neglect the holes in the concrete part of the cross-section due to steel 
bars. Thus, each bar can be described by three independent parameters: the coordinates $\left(y_{p}, z_{p}\right)$ of its centroid with respect to the coordinate system of the cross-section, and the area of the cross-section of the bar, $A_{p}$. Index $p$ takes the values from 1 to the actual number of bars, $N_{p}$. The resulting contributions to the axial force and bending moments of the cross-section due to the reinforcement are

$$
\begin{aligned}
N_{G 1 s} & =\sum_{p=1}^{N_{p}} \sigma_{s}\left(\gamma_{1}+y_{p} \kappa_{3}+z_{p} \kappa_{2}\right) A_{p} \\
M_{G 2 s} & =\sum_{p=1}^{N_{p}} z_{p} \sigma_{s}\left(\gamma_{1}+y_{p} \kappa_{3}+z_{p} \kappa_{2}\right) A_{p} \\
M_{G 3 s} & =-\sum_{p=1}^{N_{p}} y_{p} \sigma_{s}\left(\gamma_{1}+y_{p} \kappa_{3}+z_{p} \kappa_{2}\right) A_{p} .
\end{aligned}
$$

The above contributions are added to the stress resultants (9) contributed by the concrete part of the cross-section.

\subsection{Operators describing reinforced concrete}

The operators describing material of the beam, formally denoted by $\mathcal{C}_{N}$ and $\mathcal{C}_{M}$, can now be directly expressed as non-linear functions of strain vectors:

$$
\begin{aligned}
\mathcal{C}_{N} & =\left[\begin{array}{c}
N_{G 1 c}+N_{G 1 s} \\
N_{G 2 c} \\
N_{G 3 c}
\end{array}\right] \\
\mathcal{C}_{M} & =\left[\begin{array}{c}
M_{G 1 c} \\
M_{G 2 c}+M_{G 2 s} \\
M_{G 3 c}+M_{G 3 s}
\end{array}\right],
\end{aligned}
$$

where the constitutive resultant forces and moments are defined by Eqs. (9), (10), and (12). For an arbitrary strain state at the cross-section, the constitutive forces and moments can be directly evaluated from Eqs. (13)-(14). The above expressions are not invertible, which makes the direct evaluation of strains from given stresses impossible and thus has a considerable effect on the efficiency of a numerical approach.

For the iterative solution of strain vectors from constitutive equations or, alternatively, for an iterative solution of the governing equations of the beam, we also need to calculate the tangent constitutive matrix of the cross-section. 
We differentiate Eqs. (13)-(14) with respect to the strain vector components:

$$
\begin{aligned}
\frac{\partial \mathcal{C}_{N 1}}{\partial \gamma_{1}} & =\iint_{\mathcal{A}} \frac{d \sigma}{d \varepsilon} d y d z+\sum_{p=1}^{N_{p}} \frac{d \sigma_{s}}{d \varepsilon} A_{p} \\
\frac{\partial \mathcal{C}_{N 2}}{\partial \gamma_{2}} & =G A_{2} \\
\frac{\partial \mathcal{C}_{N 3}}{\partial \gamma_{3}} & =G A_{3} \\
\frac{\partial \mathcal{C}_{N 1}}{\partial \kappa_{2}} & =\frac{\partial \mathcal{C}_{M 2}}{\partial \gamma_{1}}=\iint_{\mathcal{A}} z \frac{d \sigma}{d \varepsilon} d y d z+\sum_{p=1}^{N_{p}} \frac{d \sigma_{s}}{d \varepsilon} z_{p} A_{p} \\
\frac{\partial \mathcal{C}_{N 1}}{\partial \kappa_{3}} & =\frac{\partial \mathcal{C}_{M 3}}{\partial \gamma_{1}}=\iint_{\mathcal{A}} y \frac{d \sigma}{d \varepsilon} d y d z+\sum_{p=1}^{N_{p}} \frac{d \sigma_{s}}{d \varepsilon} y_{p} A_{p} \\
\frac{\partial \mathcal{C}_{M 1}}{\partial \kappa_{1}} & =G J_{t} \\
\frac{\partial \mathcal{C}_{M 2}}{\partial \kappa_{2}} & =\iint_{\mathcal{A}} z^{2} \frac{d \sigma}{d \varepsilon} d y d z+\sum_{p=1}^{N_{p}} \frac{d \sigma_{s}}{d \varepsilon} z_{p}^{2} A_{p} \\
\frac{\partial \mathcal{C}_{M 2}}{\partial \kappa_{3}} & =\frac{\partial \mathcal{C}_{M 3}}{\partial \kappa_{2}}=\iint_{\mathcal{A}} y z \frac{d \sigma}{d \varepsilon} d y d z+\sum_{p=1}^{N_{p}} \frac{d \sigma_{s}}{d \varepsilon} y_{p} z_{p} A_{p} \\
\frac{\partial \mathcal{C}_{M 3}}{\partial \kappa_{3}} & =\iint_{\mathcal{A}} y^{2} \frac{d \sigma}{d \varepsilon} d y d z+\sum_{p=1}^{N_{p}} \frac{d \sigma_{s}}{d \varepsilon} y_{p}^{2} A_{p} .
\end{aligned}
$$

The remaining derivatives are zero. The partial derivatives of $\mathcal{C}_{N}$ and $\mathcal{C}_{M}$ with respect to $\gamma_{G}$ and $\kappa_{G}$ are stored in four matrices

$$
\begin{array}{ll}
\mathbf{C}_{\gamma \gamma}=\left[\frac{\partial \mathcal{C}_{N i}}{\partial \gamma_{j}}\right], & \mathbf{C}_{\gamma \kappa}=\left[\frac{\partial \mathcal{C}_{N i}}{\partial \kappa_{j}}\right] \\
\mathbf{C}_{\kappa \gamma}=\left[\frac{\partial \mathcal{C}_{M i}}{\partial \gamma_{j}}\right], & \mathbf{C}_{\kappa \kappa}=\left[\frac{\partial \mathcal{C}_{M i}}{\partial \kappa_{j}}\right],
\end{array}
$$

representing the block components of the cross-section constitutive tangent matrix:

$$
\mathbf{C}=\left[\begin{array}{ll}
\mathbf{C}_{\gamma \gamma} & \mathbf{C}_{\gamma \kappa} \\
\mathbf{C}_{\kappa \gamma} & \mathbf{C}_{\kappa \kappa}
\end{array}\right] .
$$

\section{Various approaches for solving constitutive equations}

When solving the governing equations of the beam, the constitutive equations (1)-(2) can be treated in several ways. Three essentially different approaches are analyzed in the paper: 
i) the direct global approach, where the constitutive stress-resultant force and moment vectors are evaluated directly from the known strains in each step of the global iteration;

ii) the local or indirect global approach, where the strain vectors are obtained iteratively from the equilibrium stress-resultant force and moment vectors in each step of the global iteration;

iii) the partly reduced approach, where only the constitutive equation for forces is eliminated from the system of the governing equations.

A short outline of each approach and its numerical implementation is given first. Then the comparisons are presented showing the convergence rates and the overall computational cost.

\subsection{Direct global approach}

The direct global approach considers the constitutive equations (1)-(2) as members of the equations of the beam on equal terms. Observe that Eqs. (1) and (2) require the cross-sectional force and moment resultants, as computed from the constitutive equations, to be equal to the force and moment vectors satisfying the equilibrium equations (3)-(4). We call this requirement the 'consistent equilibrium at the cross-section'. The satisfaction of these equations avoids a substantial source of error in materially non-linear problems as discussed and quantified in the elastic-plastic finite element analysis of plane frames in Vratanar and Saje [10].

Since the governing equations (1)-(6) are non-linear, they must be solved iteratively using, e.g. Newton's method. The differential equations under consideration are generally too complicated for the analytical solution to be possible; therefore, the finite-element discretization needs to be done. The crucial part of any finite-element formulation is the choice of primary variables and their interpolation. We here assume that strain measures are primary interpolated variables [11], [12], [13]. The displacement and rotational vectors need not be interpolated (since they can be expressed from the interpolated strains). Note that the direct consideration of the two constitutive equations (1)-(2) as equal members of the overall beam equations requires the interpolation of 6 unknown scalar functions. Thus, both strain vectors, $\boldsymbol{\gamma}_{G}(x)$ and $\boldsymbol{\kappa}_{G}(x)$, are replaced by a set of their unknown values $\boldsymbol{\gamma}_{G}^{q}$ and $\boldsymbol{\kappa}_{G}^{q}$ at discrete points $x_{q} ; q=1, \ldots, N$, chosen from the interval $[0, L]$, and interpolated by a set of $N$ interpolation functions $I_{q}(x)$

$$
\begin{aligned}
& \boldsymbol{\gamma}_{G}(x)=I_{q}(x) \boldsymbol{\gamma}_{G}^{q} \\
& \boldsymbol{\kappa}_{G}(x)=I_{q}(x) \boldsymbol{\kappa}_{G}^{q}
\end{aligned}
$$

The equilibrium and the kinematic equations (3)-(6) are exactly satisfied, the 
constitutive equations (1)-(2) are here, however, satisfied only at the interpolation points $x_{q} ; q=1, \ldots, N$ (collocation-type of discretization). After such a finite-element discretization is made, a set of $2 N+6$ non-linear algebraic matrix equations is obtained, which needs to be solved for $2 N+6$ unknowns $\boldsymbol{r}_{g}^{0}, \boldsymbol{r}_{g}^{L}, \boldsymbol{\vartheta}_{g}^{0}, \boldsymbol{\vartheta}_{g}^{L}, \boldsymbol{N}_{g}^{0}, \boldsymbol{M}_{g}^{0}, \boldsymbol{\gamma}_{G}^{q}$ and $\boldsymbol{\kappa}_{G}^{q}(q=1, \ldots, N)$ :

$$
\begin{aligned}
\boldsymbol{f}_{p} & =\mathbf{R}\left(x_{q}\right) \mathcal{C}_{N}\left(\gamma_{G}\left(x_{q}\right), \boldsymbol{\kappa}_{G}\left(x_{q}\right)\right)-\boldsymbol{N}_{g}\left(x_{q}\right)=\mathbf{0} \\
\boldsymbol{f}_{N+p} & =\mathbf{R}\left(x_{q}\right) \mathcal{C}_{M}\left(\gamma_{G}\left(x_{q}\right), \boldsymbol{\kappa}_{G}\left(x_{q}\right)\right)-\boldsymbol{M}_{g}\left(x_{q}\right)=\mathbf{0} \\
\boldsymbol{f}_{2 N+1} & =\boldsymbol{r}_{g}^{L}-\boldsymbol{r}_{g}^{0}-\int_{0}^{L} \mathbf{R}\left(\boldsymbol{\gamma}_{G}-\boldsymbol{c}_{G}\right) d x=\mathbf{0} \\
\boldsymbol{f}_{2 N+2} & =\boldsymbol{\vartheta}_{g}^{L}-\boldsymbol{\vartheta}_{g}^{0}-\int_{0}^{L} \mathbf{T}^{-T}\left(\boldsymbol{\vartheta}_{g}\right)\left(\boldsymbol{\kappa}_{G}-\boldsymbol{d}_{G}\right) d x=\mathbf{0} \\
\boldsymbol{f}_{2 N+3} & =\boldsymbol{S}_{g}^{0}+\boldsymbol{N}_{g}^{0}=\mathbf{0} \\
\boldsymbol{f}_{2 N+4} & =\boldsymbol{P}_{g}^{0}+\boldsymbol{M}_{g}^{0}=\mathbf{0} \\
\boldsymbol{f}_{2 N+5} & =\boldsymbol{S}_{g}^{L}-\boldsymbol{N}_{g}^{0}+\int_{0}^{L} \boldsymbol{n}_{g} d x=\mathbf{0} \\
\boldsymbol{f}_{2 N+6} & =\boldsymbol{P}_{g}^{L}-\boldsymbol{M}_{g}^{0}-\int_{0}^{L}\left[\boldsymbol{N}_{g} \times \mathbf{R}\left(\boldsymbol{\gamma}_{G}-\boldsymbol{c}_{G}\right)-\boldsymbol{m}_{g}\right] d x=\mathbf{0} .
\end{aligned}
$$

Following Newton's iteration scheme, a system of linear equations is solved at each iteration step $n=0,1,2, \ldots$

$$
\mathbf{K}\left(\boldsymbol{y}^{[n]}\right) \delta \boldsymbol{y}=-\boldsymbol{f}\left(\boldsymbol{y}^{[n]}\right)
$$

where $\boldsymbol{y}^{[n]}=\left[\boldsymbol{r}_{g}^{0}, \boldsymbol{r}_{g}^{L}, \boldsymbol{\vartheta}_{g}^{0}, \boldsymbol{\vartheta}_{g}^{L}, \boldsymbol{N}_{g}^{0}, \boldsymbol{M}_{g}^{0}, \boldsymbol{\gamma}_{G}^{q}, \boldsymbol{\kappa}_{G}^{q}\right]^{T}$ is the iterative vector of unknown discrete values, $\boldsymbol{f}^{[n]}$ the residual vector, $\mathbf{K}^{[n]}=\left[\frac{\partial f_{i}}{\partial y_{j}}\right]_{i, j=1 \ldots 6 N+18}$ is the global Jacobian tangent matrix , and $\delta \boldsymbol{y}$ a vector of corrections. The algorithm is presented in Box 1. 
Box 1

Direct global approach.

Gathering the required data (loading, material parameters, ...).

Determination of $\boldsymbol{r}_{g}^{0[0]}, \boldsymbol{r}_{g}^{L[0]}, \boldsymbol{\vartheta}_{g}^{0[0]}, \boldsymbol{\vartheta}_{g}^{L[0]}, \boldsymbol{N}_{g}^{0[0]}, \boldsymbol{M}_{g}^{0[0]}, \boldsymbol{\gamma}_{G}^{q[0]}, \boldsymbol{\kappa}_{G}^{q[0]}$

(from initial geometry).

Start of Newton's iterations $n=0,1,2, \ldots$

Evaluate $\boldsymbol{f}\left(\boldsymbol{y}^{[n]}\right)$ and $\mathbf{K}\left(\boldsymbol{y}^{[n]}\right)$ (Eqs. (29)-(36) and their derivatives).

Solve the system $\mathbf{K}\left(\boldsymbol{y}^{[n]}\right) \delta \boldsymbol{y}=-\boldsymbol{f}\left(\boldsymbol{y}^{[n]}\right)$ for $\delta \boldsymbol{y}$.

Update the value of $\boldsymbol{y}^{[n]}$ using $\delta \boldsymbol{y}$.

Continue iterations until $\left\|\boldsymbol{f}\left(\boldsymbol{y}^{[n]}\right)\right\| \leq \delta_{1}$ and $\|\delta \boldsymbol{y}\| \leq \delta_{2}$.

The description of the implementation of such a finite-element formulation is beyond the scope of this article (for the details, see [12]). Since the constitutive equations are taken as being parts of the global governing equations, the size of the global system of equations is large. The advantage is that the constitutive equations are automatically satisfied once the global iteration is completed. Because we do not need to solve the non-linear material equations with full precision in each global iteration step, the overall computational cost of the algorithm could however be lower. The efficiency of the direct global approach will be demonstrated in numerical examples.

\subsection{Indirect global approach}

Here we assume that the constitutive equations (1)-(2) are formally taken in their inverse form. By rearranging the terms in Eqs. (1)-(2), the constitutive equations can be put into the form appropriate for the evaluation of strains from the known equilibrium forces and moments:

$$
\begin{aligned}
& \mathcal{C}_{N}\left(\boldsymbol{\gamma}_{G}(x), \boldsymbol{\kappa}_{G}(x)\right)=\mathbf{R}^{T}(x) \boldsymbol{N}_{g}(x) \\
& \mathcal{C}_{M}\left(\boldsymbol{\gamma}_{G}(x), \boldsymbol{\kappa}_{G}(x)\right)=\mathbf{R}^{T}(x) \boldsymbol{M}_{g}(x)
\end{aligned}
$$

Eqs. (38) and (39) are non-linear algebraic equations for $\boldsymbol{\gamma}_{G}$ and $\boldsymbol{\kappa}_{G}$ and must be solved iteratively. As the strain vectors are now obtained from the constitutive equations (38)-(39), they will be termed the constitutive strains and denoted by upper index $C$, i.e. $\gamma_{G}^{C}$ and $\boldsymbol{\kappa}_{G}^{C}$. 
On the other hand, the strains can also be obtained from displacements and rotations and are thus related to the kinematic vectors, see Eqs. (5)-(6). These strains are called the kinematic strains. The two kinds of strain vectors should theoretically be the same. This is, however, not the case in the discretized solution, unless the condition is explicitly required to be satisfied. In the indirect global approach, we will explicitly require the equality of constitutive and kinematic strains :

$$
\begin{aligned}
& \gamma_{G}^{C}(x)-\boldsymbol{\gamma}_{G}(x)=\mathbf{0} \\
& \boldsymbol{\kappa}_{G}^{C}(x)-\boldsymbol{\kappa}_{G}(x)=\mathbf{0} .
\end{aligned}
$$

As in the direct approach, we interpolate the kinematic strain vectors $\gamma_{G}(x)$ and $\boldsymbol{\kappa}_{G}(x)$ and discretize Eqs. (40)-(41) to be satisfied at interpolation points $x_{q} ; q=1, \ldots, N$. The final set of the governing equations of the beam is much like but not equal to the one for the direct approach, and reads:

$$
\begin{aligned}
\boldsymbol{h}_{p} & =\gamma_{G}^{C}\left(x_{q}\right)-\gamma_{G}\left(x_{q}\right)=\mathbf{0} \\
\boldsymbol{h}_{N+p} & =\boldsymbol{\kappa}_{G}^{C}\left(x_{q}\right)-\boldsymbol{\kappa}_{G}\left(x_{q}\right)=\mathbf{0} \\
\boldsymbol{h}_{2 N+1} & =\boldsymbol{r}_{g}^{L}-\boldsymbol{r}_{g}^{0}-\int_{0}^{L} \mathbf{R}\left(\boldsymbol{\gamma}_{G}-\boldsymbol{c}_{G}\right) d x=\mathbf{0} \\
\boldsymbol{h}_{2 N+2} & =\boldsymbol{\vartheta}_{g}^{L}-\boldsymbol{\vartheta}_{g}^{0}-\int_{0}^{L} \mathbf{T}^{-T}\left(\boldsymbol{\vartheta}_{g}\right)\left(\boldsymbol{\kappa}_{G}-\boldsymbol{d}_{G}\right) d x=\mathbf{0} \\
\boldsymbol{h}_{2 N+3} & =\boldsymbol{S}_{g}^{0}+\boldsymbol{N}_{g}^{0}=\mathbf{0} \\
\boldsymbol{h}_{2 N+4} & =\boldsymbol{P}_{g}^{0}+\boldsymbol{M}_{g}^{0}=\mathbf{0} \\
\boldsymbol{h}_{2 N+5} & =\boldsymbol{S}_{g}^{L}-\boldsymbol{N}_{g}^{0}+\int_{0}^{L} \boldsymbol{n}_{g} d x=\mathbf{0} \\
\boldsymbol{h}_{2 N+6} & =\boldsymbol{P}_{g}^{L}-\boldsymbol{M}_{g}^{0}-\int_{0}^{L}\left[\boldsymbol{N}_{g} \times \mathbf{R}\left(\boldsymbol{\gamma}_{G}-\boldsymbol{c}_{G}\right)-\boldsymbol{m}_{g}\right] d x=\mathbf{0} .
\end{aligned}
$$

As the constitutive equations are not invertible, the constitutive strains can not be directly obtained, which results in an algorithm essentially different compared with the one for the direct global approach. The algorithm is displayed in Box 2.

The size of the system of the overall equations is thus not changed. The numerical solution is obtained much as in [12], with an important exception, however, that the consistency equations (38)-(39) now need to be solved to final precision within each step of the global iteration. Certainly, this could increase the overall computational time. We will also investigate a simplified global iteration process, in which Eqs. (38)-(39) are solved only approximately by using a single step of local Newton's iteration. 
Box 2

Indirect global approach.

Gathering the required data (loading, material parameters, ...).

Determination of $\boldsymbol{r}_{g}^{0[0]}, \boldsymbol{r}_{g}^{L[0]}, \boldsymbol{\vartheta}_{g}^{0[0]}, \boldsymbol{\vartheta}_{g}^{L[0]}, \boldsymbol{N}_{g}^{0[0]}, \boldsymbol{M}_{g}^{0[0]}, \boldsymbol{\gamma}_{G}^{q[0]}, \boldsymbol{\kappa}_{G}^{q[0]}$

(from initial geometry).

Start of Newton's iterations $n=0,1,2, \ldots$

Evaluate $\boldsymbol{N}_{g}^{[n]}\left(x_{q}\right)$ and $\boldsymbol{M}_{g}^{[n]}\left(x_{q}\right)$ (Eqs. (3)-(4)).

Set the initial values for constitutive strains $\gamma_{G}^{C q[0]}=\gamma_{G}^{q[n]}, \boldsymbol{\kappa}_{G}^{C q[0]}=\boldsymbol{\kappa}_{G}^{q[n]}$.

Start of local Newton's iterations $k=0,1,2, \ldots$ at each $x_{q}$.

Evaluate $\mathcal{C}_{N}\left(\boldsymbol{\gamma}_{G}^{C q[k]}, \boldsymbol{\kappa}_{G}^{C q[k]}\right)$ and $\mathcal{C}_{M}\left(\gamma_{G}^{C q[k]}, \boldsymbol{\kappa}_{G}^{C q[k]}\right)($ Eqs. (13)-(14)).

Evaluate $\mathbf{C}\left(\gamma_{G}^{C q[k]}, \boldsymbol{\kappa}_{G}^{C q[k]}\right)$ (Eq. (26)).

Solve the system $\mathbf{C}^{[k]}\left[\begin{array}{c}\delta \boldsymbol{\gamma} \\ \delta \boldsymbol{\kappa}\end{array}\right]=-\left[\begin{array}{c}\mathcal{C}_{N}^{[k]}-\mathbf{R}^{[n] T} \boldsymbol{N}_{g}^{[n]} \\ \mathcal{C}_{M}^{[k]}-\mathbf{R}^{[n] T} \boldsymbol{M}_{g}^{[n]}\end{array}\right]$ for $\delta \boldsymbol{\gamma}$ and $\delta \boldsymbol{\kappa}$.

Update the strains: $\boldsymbol{\gamma}_{G}^{C q[k+1]}=\boldsymbol{\gamma}_{G}^{C q[k]}+\delta \boldsymbol{\gamma}, \boldsymbol{\kappa}_{G}^{C q[k+1]}=\boldsymbol{\kappa}_{G}^{C q[k]}+\delta \boldsymbol{\kappa}$.

Continue iterations until $\left\|\left[\begin{array}{l}\mathcal{C}_{N}^{[k]}-\mathbf{R}^{[n] T} \boldsymbol{N}_{g}^{[n]} \\ \mathcal{C}_{M}^{[k]}-\mathbf{R}^{[n] T} \boldsymbol{M}_{g}^{[n]}\end{array}\right]\right\| \leq \delta_{3}$ and $\left\|\left[\begin{array}{c}\delta \boldsymbol{\gamma} \\ \delta \boldsymbol{\kappa}\end{array}\right]\right\| \leq \delta_{4}$.

Evaluate $\boldsymbol{h}\left(\boldsymbol{y}^{[n]}\right)$ (Eqs. (42)-(49)).

Evaluate the tangent matrix $\mathbf{K}^{[n]}=\left[\frac{\partial h_{i}}{\partial y_{j}}\right]_{i, j=1 \ldots 6 N+18}$.

Solve the system $\mathbf{K}^{[n]} \delta \boldsymbol{y}=-\boldsymbol{h}\left(\boldsymbol{y}^{[n]}\right)$ for $\delta \boldsymbol{y}$.

Update the value of $\boldsymbol{y}^{[n]}$ using $\delta \boldsymbol{y}$.

Continue iterations until $\left\|\boldsymbol{h}\left(\boldsymbol{y}^{[n]}\right)\right\| \leq \delta_{1}$ and $\|\delta \boldsymbol{y}\| \leq \delta_{2}$. 


\subsection{Partly reduced approach}

We will now eliminate Eq. (1) from the set of governing equations in order to reduce the size of the element tangent stiffness matrix. By separately performing an iterative solution of (38) at an arbitrary configuration of the beam, we are able to determine uniquely the strain vector $\boldsymbol{\gamma}$, provided that $\boldsymbol{\kappa}$ is known. The remaining Eqs. (2)-(6) are left unchanged, resulting in a lower number of global degrees of freedom. The discretization now requires the interpolation of at least three unknown scalar functions. One such formulation, that is in accord with this approach, employs the curvature vector $\boldsymbol{\kappa}_{G}(x)$ as the only interpolated variable [11]. The governing equations of such a formulation are as follows:

$$
\begin{aligned}
\boldsymbol{b}_{p} & =\mathcal{C}_{M}\left(\boldsymbol{\gamma}_{G}, \boldsymbol{\kappa}_{G}\right)-\mathbf{R}^{T} \boldsymbol{M}_{g}=\mathbf{0} \\
\boldsymbol{b}_{N+1} & =\boldsymbol{r}_{g}^{L}-\boldsymbol{r}_{g}^{0}-\int_{0}^{L} \mathbf{R}\left(\boldsymbol{\gamma}_{G}-\boldsymbol{c}_{G}\right) d x=\mathbf{0} \\
\boldsymbol{b}_{N+2} & =\boldsymbol{\vartheta}_{g}^{L}-\boldsymbol{\vartheta}_{g}^{0}-\int_{0}^{L} \mathbf{T}^{-T}\left(\boldsymbol{\vartheta}_{g}\right)\left(\boldsymbol{\kappa}_{G}-\boldsymbol{d}_{G}\right) d x=\mathbf{0} \\
\boldsymbol{b}_{N+3} & =\boldsymbol{S}_{g}^{0}+\boldsymbol{N}_{g}^{0}=\mathbf{0} \\
\boldsymbol{b}_{N+4} & =\boldsymbol{P}_{g}^{0}+\boldsymbol{M}_{g}^{0}=\mathbf{0} \\
\boldsymbol{b}_{N+5} & =\boldsymbol{S}_{g}^{L}-\boldsymbol{N}_{g}^{0}+\int_{0}^{L} \boldsymbol{n}_{g} d x=\mathbf{0} \\
\boldsymbol{b}_{N+6} & =\boldsymbol{P}_{g}^{L}-\boldsymbol{M}_{g}^{0}-\int_{0}^{L}\left[\boldsymbol{N}_{g} \times \mathbf{R}\left(\boldsymbol{\gamma}_{G}-\boldsymbol{c}_{G}\right)-\boldsymbol{m}_{g}\right] d x=\mathbf{0} .
\end{aligned}
$$


Box 3

Partly reduced approach.

Gathering the required data (loading, material parameters, ...).

Determination of $\boldsymbol{r}_{g}^{0[0]}, \boldsymbol{r}_{g}^{L[0]}, \boldsymbol{\vartheta}_{g}^{0[0]}, \boldsymbol{\vartheta}_{g}^{L[0]}, \boldsymbol{N}_{g}^{0[0]}, \boldsymbol{M}_{g}^{0[0]}, \boldsymbol{\kappa}_{G}^{q[0]}$

(from initial geometry).

Start of Newton's iterations $n=0,1,2, \ldots$

Evaluate $\boldsymbol{N}_{g}^{[n]}\left(x_{q}\right)$ (Eq. (3))

Guess the initial values for translational strains $\gamma_{G}^{q[0]}$.

Start of local Newton's iterations $k=0,1,2, \ldots$ at each $x_{q}$.

Evaluate $\mathcal{C}_{N}\left(\gamma_{G}^{q[k]}, \boldsymbol{\kappa}_{G}^{q[n]}\right)($ Eq. (13))

Evaluate $\mathbf{C}_{\gamma \gamma}\left(\boldsymbol{\gamma}_{G}^{q[k]}, \boldsymbol{\kappa}_{G}^{q[n]}\right)$ (Eq. (24)).

Solve the system $\mathbf{C}_{\gamma \gamma}^{[k]} \delta \boldsymbol{\gamma}=-\left(\mathcal{C}_{N}^{[k]}-\mathbf{R}^{[n] T} \boldsymbol{N}_{g}^{[n]}\right)$ for $\delta \boldsymbol{\gamma}$.

Update the translational strains: $\gamma_{G}^{q[k+1]}=\gamma_{G}^{q[k]}+\delta \gamma$.

Continue iterations until $\left\|\mathcal{C}_{N}^{[k]}-\mathbf{R}^{[n] T} \boldsymbol{N}_{g}^{[n]}\right\| \leq \delta_{3}$ and $\|\delta \boldsymbol{\gamma}\| \leq \delta_{4}$.

Evaluate $\boldsymbol{b}\left(\boldsymbol{z}^{[n]}\right)$ (Eqs. (50)-(56));

where $\boldsymbol{z}^{[n]}=\left[\boldsymbol{r}_{g}^{0[n]}, \boldsymbol{r}_{g}^{L[n]}, \boldsymbol{\vartheta}_{g}^{0[n]}, \boldsymbol{\vartheta}_{g}^{L[n]}, \boldsymbol{N}_{g}^{0[n]}, \boldsymbol{M}_{g}^{0[n]}, \boldsymbol{\kappa}_{G}^{q[n]}\right]^{T}$.

Evaluate the tangent matrix $\mathbf{K}^{[n]}=\left[\frac{\partial b_{i}}{\partial y_{j}}\right]_{i, j=1 \ldots 6 N+18}$.

Solve the system $\mathbf{K}^{[n]} \delta \boldsymbol{z}=-\boldsymbol{b}\left(\boldsymbol{z}^{[n]}\right)$ for $\delta \boldsymbol{z}$.

Update the value of $\boldsymbol{z}^{[n]}$ using $\delta \boldsymbol{z}$.

Continue iterations until $\left\|\boldsymbol{b}\left(\boldsymbol{z}^{[n]}\right)\right\| \leq \delta_{1}$ and $\|\delta \boldsymbol{z}\| \leq \delta_{2}$.

The details of this formulation were presented by Zupan and Saje in [11]. The linearized system of global equations in this formulation is smaller, yet the extracted equation needs to be solved with a sufficient precision locally in each iteration step. Because of the non-linearity of condition (1), it has to be solved iteratively. This local iteration process demands further computational work, which may neutralize the benefit of the smaller size of the global system 
of equations. The algorithm is presented in Box 3 . Note that the partly reduced approach represents a combination of direct and indirect global approach. By reducing the global set of equations we inherently need to solve the reduced equations locally. Thus, a part of non-linear constitutive equations is solved locally and the other part is solved globally. It would be possible to consider indirect partly reduced approach, however its difference to the indirect global approach is insignificant.

We may somewhat reduce the number of local iteration steps by taking good initial guesses for $\gamma_{G}$. In the present study, we take the values of $\gamma_{G}$ at the end of the previous global iteration. This demands storing temporary values of strain vectors $\gamma_{G}$ in the computer memory, but otherwise it does not increase the computational cost. We will also asses the reduction of the cost of the local iteration, if only a single-step local Newton's method is applied. It is very interesting and useful to observe that in such a way the final converged solution is not lost as the global set of equations needs to be solved in prescribed tolerance. The accuracy of the converged solution at global level is inherited at the local level.

\section{$5 \quad$ Numerical examples}

The influence of the proposed approaches on the rate of convergence and the overall computational cost are examined through numerical examples. For each numerical example, we will count the number of iterations and the total number of floating point operations. Note that the total number of floating point operations (flops) is an objective measure of the actual computational cost of a particular finite-element formulation.

In order to make the comparisons unbiased, the computer code for all of the formulations shares the same pre- and postprocessing algorithms and all of the common parts have been coded identically. Computer programs have been developed and tested in Matlab [9], based on the algorithms, presented in [12] and [13]. We used the same type of the finite-element discretization in all formulations. Low-order linear-strain (two-point) elements, and higher-order elements with 5 internal interpolation points were used in numerical tests. The results for higher order elements are shown for demonstration of accuracy of computation and to show the high increase of the computational costs.

We have chosen to compare the efficiency of the three formulations on realistic problems using a realistic material model. The data were obtained from well documented experimental tests of reinforced concrete beams and frames. As the differences in the computational cost between the approaches grow, if the applied loads are close to the critical values, all the tests are performed in the 
neighbourhood of the critical load.

The quadratic convergence of Newton's method was achieved in all numerical examples. The iteration was terminated when the Euclidean norm of the vector of nodal unknowns, $\|\boldsymbol{\Delta} \boldsymbol{y}\|_{2}$, and of the vector of unbalanced forces, $\|\boldsymbol{f}\|_{2}$, was less than $10^{-7}$.

\subsection{Foure's column}

Foure's column was chosen by the RILEM Technical Committee as one of the bench-mark problems for testing the computational models and computer programs for reinforced concrete structures [4]. The column was clamped at one end and subjected to an eccentric axial force at the other (Fig. 5).

The geometric and material data of the column are given in [4]:

$$
\begin{array}{lll}
L=225 \mathrm{~cm} & h=20 \mathrm{~cm} & t=15 \mathrm{~cm} \\
f_{\mathrm{m}}=3.83 \mathrm{kN} / \mathrm{cm}^{2} & E_{\mathrm{s}} \varepsilon_{\mathrm{y}}=46.5 \mathrm{kN} / \mathrm{cm}^{2} . &
\end{array}
$$

The reinforcement consisted of four steel bars with one bar being placed in each corner of the rectangular cross-section. The diameter of bars was $1.2 \mathrm{~cm}$ and the concrete cover depth was $1.4 \mathrm{~cm}$. The remaining material parameters were estimated only on the basis of the given strengths and typical values for

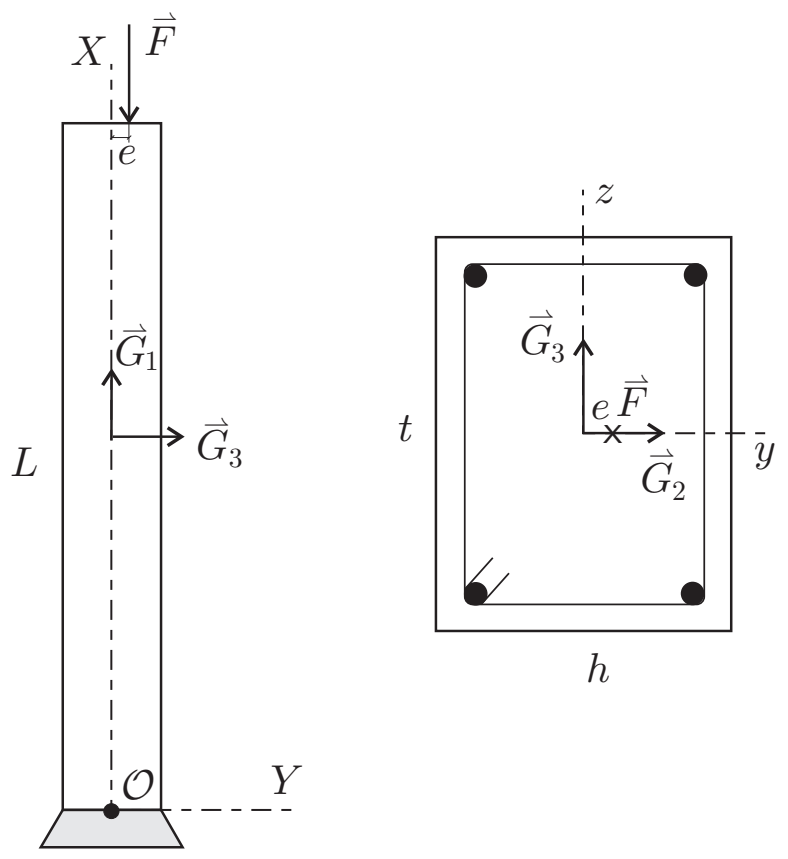

Fig. 5. Foure's column. 
the concrete and reinforcing steel:

$$
\begin{array}{llll}
\varepsilon_{\mathrm{u}}=-8 \cdot 10^{-3} & \varepsilon_{\mathrm{r}}=5.5 \cdot 10^{-5} & \varepsilon_{\mathrm{m}}=7 \cdot 10^{-4} & \varepsilon_{1}=-2.5 \cdot 10^{-3} \\
E_{\mathrm{s}}=20000 \mathrm{kN} / \mathrm{cm}^{2} & E_{\mathrm{p}}=0 & \varepsilon_{\mathrm{ym}}=0.02 . &
\end{array}
$$

Our results are shown and compared to experimental values and to the results of Bratina [2] and Kanstad [7] in Figure 5.1 we compare our results. The results compare well. As our results prove to be reliable we can now focus on convergence of the numerical algorithm.

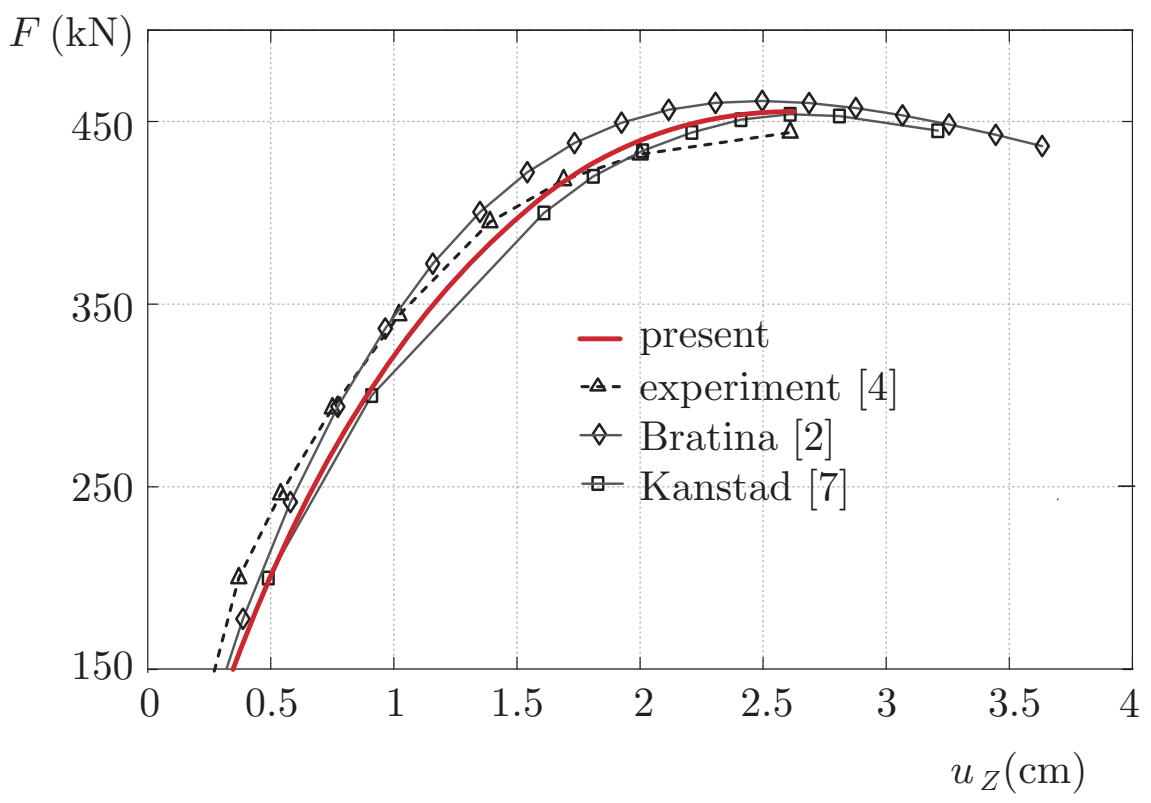

Fig. 6. Foure's column: load vs. lateral deflection.

Table 1

Convergence rates and computational costs for Foure's column [4] using two linear elements.

\begin{tabular}{lcccc}
\hline method & global iterations & local iterations & flops & lateral deflection \\
\hline direct global & 9 & - & 481292 & 2.483 \\
indirect global & 9 & $1-4$ & 657404 & 2.483 \\
indirect global, 1 it & 10 & 1 & 650830 & 2.483 \\
partly reduced & 9 & 5 & 738676 & 2.483 \\
partly reduced, 1 it & 9 & 1 & 613209 & 2.483 \\
\hline
\end{tabular}

1 it $=$ modified method where a single step in local iteration is applied,

flops $=$ total number of floating point operations. 
The comparisons were made for the axial load $F=455 \mathrm{kN}$ with eccentricity $e=1.5 \mathrm{~cm}$. In order to test the formulations in the most severe situation, the applied load was chosen to be only a little less than the calculated critical load. Results are compared in Tables 1 and 2 in terms of the number of iterations, the number of floating point operations and the free-end lateral deflection.

The comparisons of five different formulations are presented in Table 1. Two linear elements were employed to model the column. Surprisingly, the number of global iterations is the same for all formulations with a small exception for the indirect global approach with a single local iteration. On the other hand, the number of floating point operations (flops) differs substantially. When the constitutive equations are parts of the governing global equations, the overall computational cost is the lowest of all (481292 floating point operations). The direct evaluation of the material equations at the element level reduces the number of numerical operations for composing the tangent stiffness matrix of each element. Despite the larger size of the element tangent stiffness matrix, its generation demands rather small computational times. Thus, although a larger system of equations must be solved, the total number of operations is still substantially lower than for the partly reduced approach with a single local iteration (613209). In the indirect global approach and the partly reduced approach, the non-linear constitutive equations must be iteratively solved with a sufficient precision at each step of the global iteration. One to four local iterations are needed when using the indirect global formulation, and 5 in the partly reduced approach. These local iterations have to be carried out at each node of each element at each step and thus increase the computational cost of the algorithm. The cost can be reduced, if the non-linear equations are approximated by their linear forms, so that only one step of the local iteration is carried out. For the partly reduced approach, such a single-step local iteration

Table 2

Convergence rates and computational costs for Foure's column [4] using two higherorder elements with 5 interpolation points.

\begin{tabular}{lcccc}
\hline method & global iterations & local iterations & flops & lateral deflection \\
\hline direct global & 9 & - & 1510866 & 2.501 \\
indirect global & 10 & $1-4$ & 2461130 & 2.501 \\
indirect global, 1 it & 10 & 1 & 2291654 & 2.501 \\
partly reduced & 9 & 5 & 1918859 & 2.501 \\
partly reduced, 1 it & 9 & 1 & 1603198 & 2.501 \\
\hline
\end{tabular}

1 it $=$ modified method where a single step of local iteration is applied,

flops $=$ total number of floating point operations. 
reduces the computational cost from 738676 to 613209 operations. As similar behaviour is observed for the indirect global approach, yet in this case the number of global iterations appears to be higher when a single local iteration is used.

If we use higher-order elements, the number of floating point operations increases rapidly. Table 2 presents the results for the mesh of two 5-node elements. Again the global direct approach proves to be the most efficient. For example, the difference in the computational cost between the global direct approach and the partly reduced approach with iterations is $27 \%$. The efficiency of the partly reduced approach is increased, if the number of local iterations is limited to 1 ; then the computational cost becomes comparable to the direct global method.

Note that all formulations predict the same value of the lateral deflection. This confirms that our test was unbiased. The computational costs are, on the other hand, remarkably different, which suggests that finding the most suitable formulation makes sense.

\subsection{Concrete columns subjected to biaxial bending}

We analyze several short reinforced concrete columns $(L=120 \mathrm{~cm})$ subjected to eccentric axial forces at both ends. The supports at both ends allow rotations and one support additionally allows displacements in $X$ (axial) direction. The double eccentricity of the load leads to biaxial bending of the column. These columns were tested by Kim and Lee [8].

The columns have rectangular cross-sections with $h=10 \mathrm{~cm}$ and $t=20 \mathrm{~cm}$. The material properties of concrete and steel are

$$
f_{\mathrm{m}}=2.7 \mathrm{kN} / \mathrm{cm}^{2} \quad E_{\mathrm{s}} \varepsilon_{\mathrm{y}}=43.6 \mathrm{kN} / \mathrm{cm}^{2} .
$$

Reinforcing steel bars of diameter $0.95 \mathrm{~cm}$ are covered with the $2.3 \mathrm{~cm}$ thick layer of concrete. The eccentricity of the applied force is $4 \mathrm{~cm}$; the angle of eccentricity $(\alpha)$ varied from 0 to $\pi / 2$. We here analyze only one eccentricitie $\alpha=\pi / 6$. The load very close to the critical force was chosen. The following data were employed:

$$
\begin{array}{llll}
\varepsilon_{\mathrm{u}}=-8 \cdot 10^{-3} & \varepsilon_{1}=-2 \cdot 10^{-3} & \varepsilon_{\mathrm{r}}=5.5 \cdot 10^{-5} & \varepsilon_{\mathrm{m}}=7 \cdot 10^{-4} \\
E_{\mathrm{s}}=20000 \mathrm{kN} / \mathrm{cm}^{2} & E_{\mathrm{p}}=0 & \varepsilon_{\mathrm{ym}}=0.02 .
\end{array}
$$

For angle $\alpha=\pi / 6$ experimental results are available (see Figure 8). The dispersion between the two experimental results of the same case is up to 


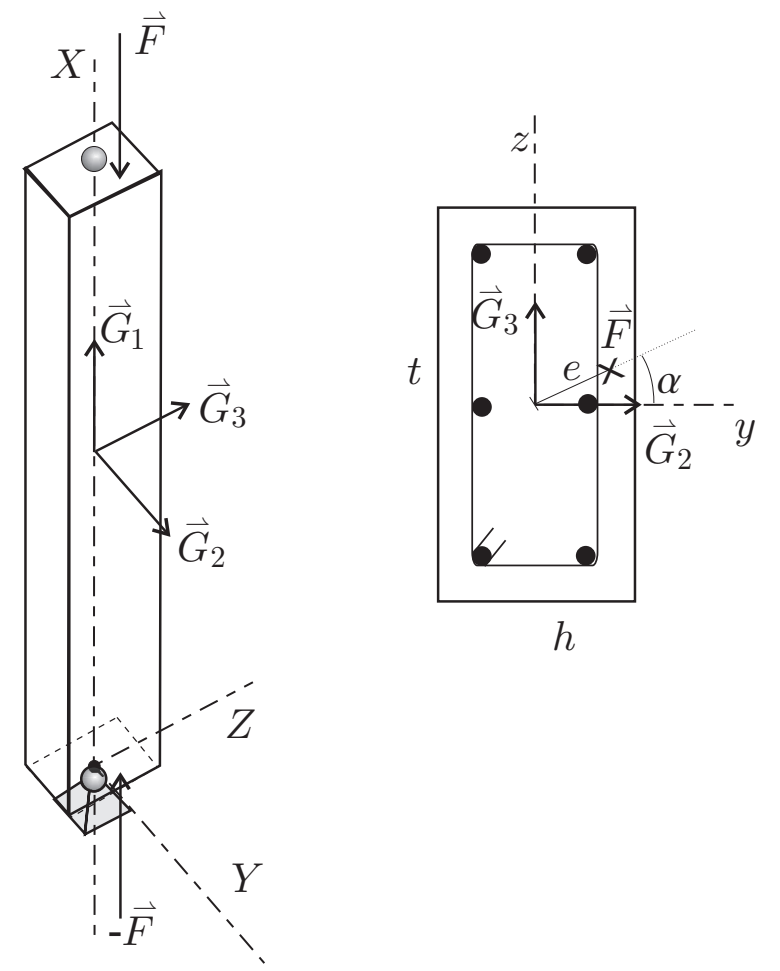

Fig. 7. Eccentrically axially loaded reinforced concrete column.

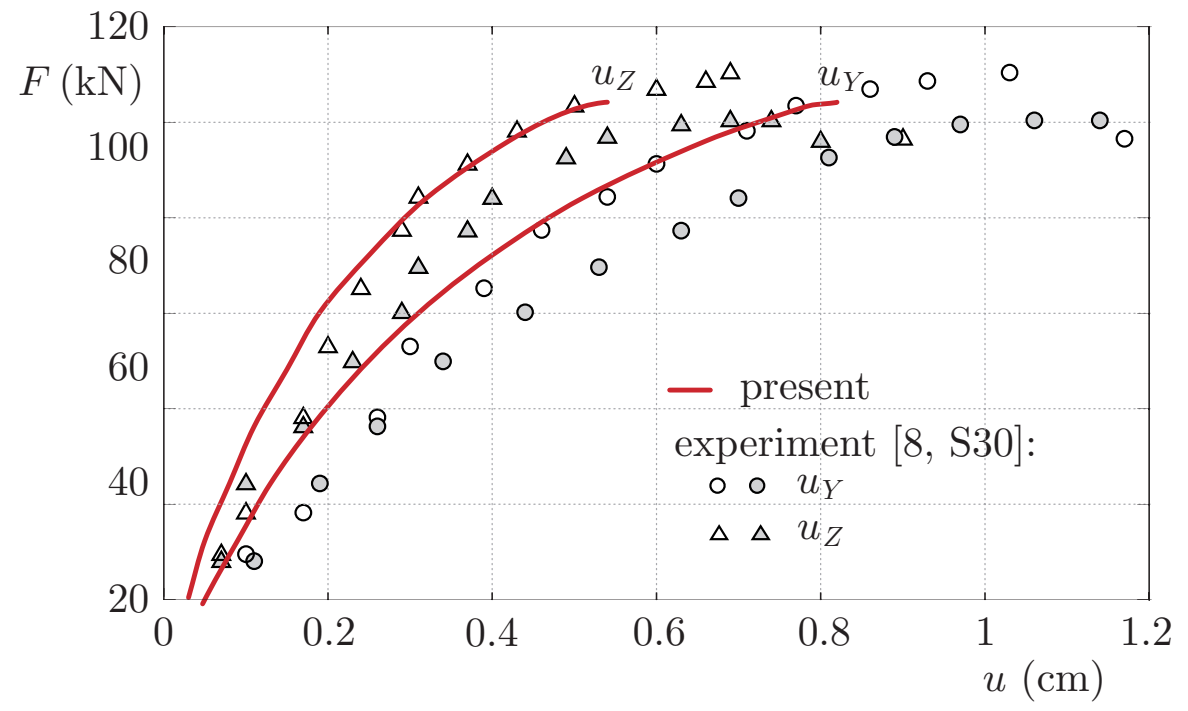

Fig. 8. Column $S 30$ : load vs. lateral deflection.

$10 \%$. With regard to the dispersion of the measured values, we find our results sufficiently accurate, which is evident from Figure 8.

Results for axial force $F=210 \mathrm{kN}$ and eccentricity $\alpha=\pi / 6$ are compared in Tables 3 and 4 in terms of the number of iterations, the number of floating 
point operations and the midpoint displacements. In this example the direct global method is particularly efficient regarding the computational cost.

Within the equal element order, the numerical results for $u_{Y}$ and $u_{Z}$ agree completely. Only a small improvement is observed, if the order of finite elements is increased from 1 to 4 .

Table 3

Convergence rates and computational costs using two linear elements for the column tested by Kim and Lee [8]. Eccentricity angle $\alpha=\pi / 6$.

\begin{tabular}{lccccc}
\hline method & global iterations & local iterations & flops & $u_{Y}$ & $u_{Z}$ \\
\hline direct global & 7 & - & 512595 & 0.794 & 0.107 \\
indirect global & 7 & $1-5$ & 667272 & 0.794 & 0.107 \\
indirect global, 1 it & 8 & 1 & 658199 & 0.794 & 0.107 \\
partly reduced & 7 & $3-5$ & 750611 & 0.794 & 0.107 \\
partly reduced, 1 it & 8 & 1 & 724465 & 0.794 & 0.107 \\
\hline
\end{tabular}

$1 \mathrm{it}=$ modified method where a single step of local iteration is applied,

flops $=$ total number of floating point operations.

Table 4

Convergence rates and computational costs using two 5-node elements for the column tested by Kim and Lee [8]. Eccentricity angle $\alpha=\pi / 6$.

\begin{tabular}{lccccc}
\hline method & global iterations & local iterations & flops & $u_{Y}$ & $u_{Z}$ \\
\hline direct global & 7 & - & 1346473 & 0.793 & 0.107 \\
indirect global & 7 & $1-5$ & 1944579 & 0.793 & 0.107 \\
indirect global, 1 it & 8 & 1 & 2003135 & 0.793 & 0.107 \\
partly reduced & 7 & $3-5$ & 1745037 & 0.793 & 0.107 \\
partly reduced, 1 it & 8 & 1 & 1705030 & 0.793 & 0.107 \\
\hline
\end{tabular}

1 it $=$ modified method where a single step of local iteration is applied,

flops $=$ total number of floating point operations. 


\subsection{Concrete frame of Ferguson and Breen}

Our last example is the $L_{3}$ frame, tested by Ferguson and Breen [5] and numerically analyzed by Bratina [2] and Gunnin et al. [6]. The dimensions of the square opening in the reinforced concrete frame is $d=203.2 \mathrm{~cm}$ (see Fig. 9).

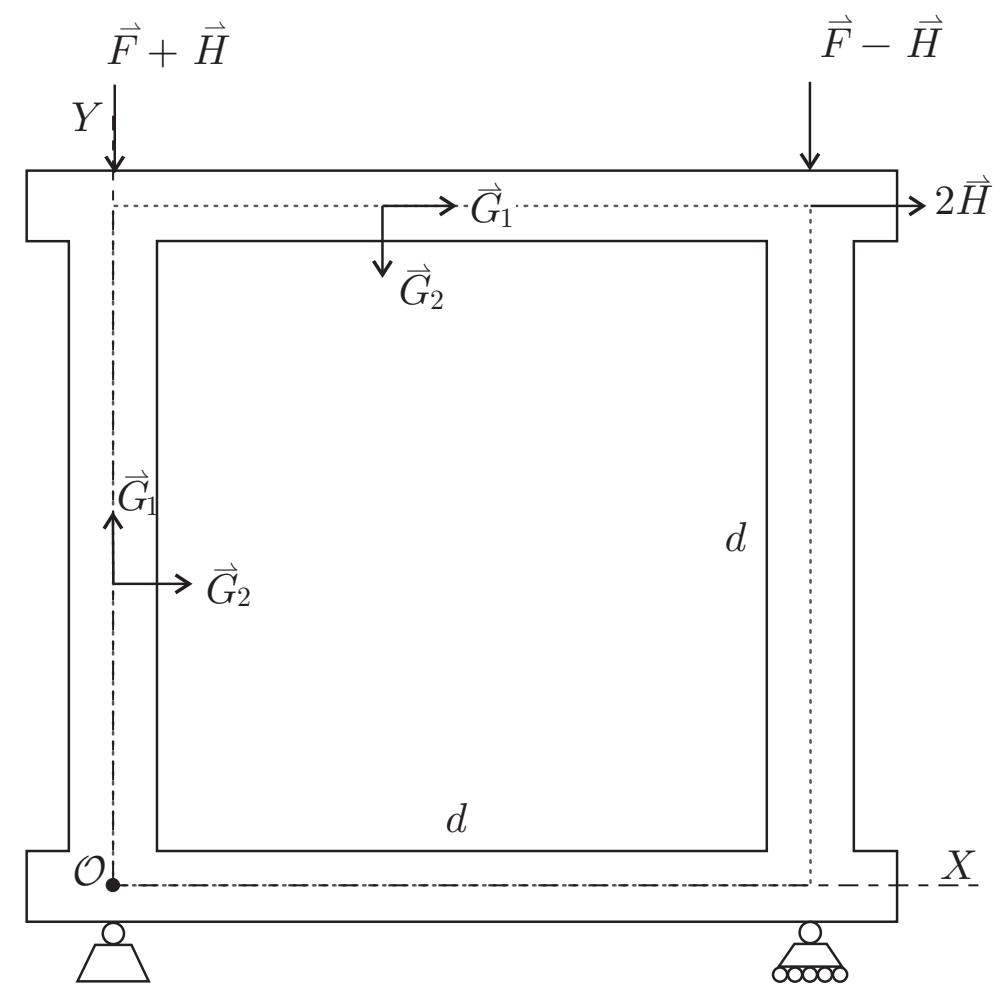

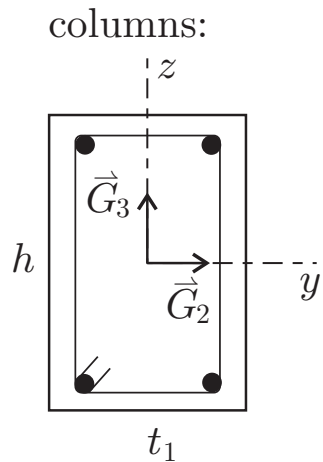

beams:

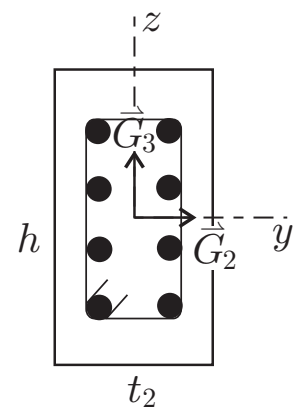

Fig. 9. $L_{3}$ frame tested by Ferguson and Breen [5].

The cross-sectional properties of columns and beams are displayed in Fig. 9. The sides of the rectangular cross-section of the columns are $h=15.2 \mathrm{~cm}, t_{1}=$ $10.2 \mathrm{~cm}$. The bars of diameter $1 \mathrm{~cm}$ are placed in each corner of the crosssection. The centroid of the first bar has the coordinates $\left(y_{p}, z_{p}\right)=(3.4,6.1)$, with the other three bars being located symmetrically according to the local coordinate axes $y$ and $z$. The sides of the beam cross-section are $h=15.2 \mathrm{~cm}$, $t_{2}=8.1 \mathrm{~cm}$. The cross-section is reinforced with eight bars having the diameter $1.3 \mathrm{~cm}$. The bars are located symmetrically according to the local coordinate axes $y$ and $z$ (Fig. 9), thus only two coordinate pairs need to be stated:

$$
\begin{aligned}
& \left(y_{p 1}, z_{p 1}\right)=(1.8,1.6) \\
& \left(y_{p 2}, z_{p 2}\right)=(1.8,4.7) .
\end{aligned}
$$

Strength of concrete in compression was in [5] given to be $f_{\mathrm{m}}=2.21 \mathrm{kN} / \mathrm{cm}^{2}$. The yield stress of steel in columns was $E_{\mathrm{s}} \varepsilon_{\mathrm{y}}=38.89 \mathrm{kN} / \mathrm{cm}^{2}$ and in the 
beams $E_{\mathrm{s}} \varepsilon_{\mathrm{y}}=40.34 \mathrm{kN} / \mathrm{cm}^{2}$. The following values of the remaining material parameters were assumed in the calculations:

$$
\begin{array}{llll}
\varepsilon_{\mathrm{u}}=-8 \cdot 10^{-3} & \varepsilon_{\mathrm{r}}=5.5 \cdot 10^{-5} & \varepsilon_{\mathrm{m}}=7 \cdot 10^{-4} & \varepsilon_{1}=-2.2 \cdot 10^{-3} \\
E_{\mathrm{s}}=20000 \mathrm{kN} / \mathrm{cm}^{2} & E_{\mathrm{p}}=0 & \varepsilon_{\mathrm{ym}}=0.02 & F=140 \mathrm{kN} .
\end{array}
$$

The loading arrangement is illustrated in Fig. 9, where $H=0.01 F$ is a small perturbation force.

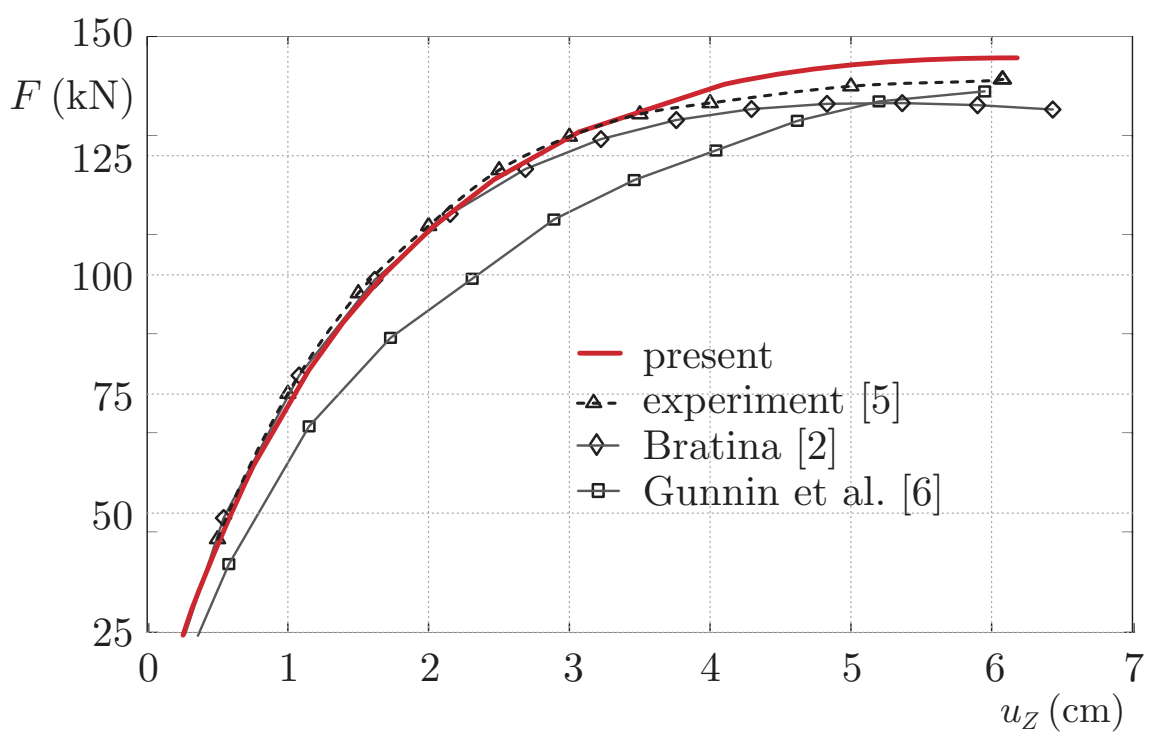

Fig. 10. Load vs. deflection of the upper right corner of $L 3$ frame.

The vertical force $F$ is increased to the critical value and the deflections of the upper right corner are analyzed. In Figure 10 we show the comparison of the present results to the experimental data and to numerical results of Bratina [2] and Gunnin et al. [6]. Our results are in good agreement with the experiment and other authors.

The computational costs and the deflections of the upper right corner for different formulations are compared in Tables 5 and 6. Again, the direct global approach requires the lowest number of operations. For this numerical example, the number of global iterations is eight for both the direct and indirect global approach when a high order element is used. 
Table 5

Convergence rates and computational costs for $L_{3}$ frame using two linear elements.

\begin{tabular}{lcccc}
\hline method & global iterations & local iterations & flops & $u_{Z}$ \\
\hline direct global & 7 & - & 1464768 & 3.805 \\
indirect global & 7 & $1-4$ & 1979577 & 3.805 \\
indirect global, 1 it & 8 & 1 & 2059817 & 3.805 \\
partly reduced & 7 & $4-5$ & 2132472 & 3.805 \\
partly reduced, 1 it & 8 & 1 & 2060391 & 3.805 \\
\hline
\end{tabular}

1 it $=$ modified method where a single step of local iteration is applied, flops $=$ total number of floating point operations.

Table 6

Convergence rates and computational costs for $L_{3}$ frame using two elements with 5 internal nodes.

\begin{tabular}{lcccc}
\hline method & global iterations & local iterations & flops & $u_{X}$ \\
\hline direct global & 8 & - & 5401430 & 3.916 \\
indirect global & 8 & $1-4$ & 6816894 & 3.916 \\
indirect global, 1 it & 8 & 1 & 7330265 & 3.916 \\
partly reduced & 7 & $4-5$ & 5672299 & 3.916 \\
partly reduced, 1 it & 8 & 1 & 5584964 & 3.916 \\
\hline
\end{tabular}

1 it $=$ modified method where a single step of local iteration is applied,

flops $=$ total number of floating point operations.

\section{Conclusions}

We investigated how various approaches for considering the non-linear constitutive equations of the non-linear spatial beam formulations effect the rate of the global convergence and the total number of floating point operations. The essential results of the study are:

(i) For a chosen finite-element mesh and interpolation, the numerical results agree completely for any approach.

(ii) The computational costs are remarkably different. 
(iii) The global approach does not increase the number of global iterations.

(iv) A higher number of global iterations occurs in the reduced approach, if a single local iteration is used. The computational cost are however much lower, as if we fully iterate at the local lever.

(v) In all numerical examples considered here, the direct global approach required the smallest number of floating point operations. Thus, the direct global approach seems to be optimal for solving the equations of the beam.

\section{References}

[1] P. G. Bergan, I. Holand, "Nonlinear finite element analysis of concrete structures", Comput. Methods Appl. Mech. Engrg. 17/18, 443-467, 1979.

[2] S. Bratina, "Fire-response analysis of reinforced concrete plane frames" (in Slovene), PhD Thesis, University of Ljubljana, Faculty of Civil and Geodetic Engineering, Ljubljana, 2003.

[3] P. Desayi, S. Krishnan, "Equation for the stress-strain curve of concrete", J. Am. Concrete Inst. 61, 345-350, 1964.

[4] B. Espion, "Benchmark examples for creep and shrinkage analysis computer programs", Creep and shrinkage of concrete, RILEM TC 114 subcommittee 3, 1993.

[5] P. M. Ferguson, J. E. Breen, "Investigation of the long concrete column in a frame subject to lateral loads", Symp. On Reinforced Concrete Columns, ACI SP-13, 75-119, 1966.

[6] B. L. Gunnin, F. N. Rad, R. W. Furlong, "A general nonlinear analysis of concrete structures and comparison with frame tests", Comput. Struct. 7, 257$265,1977$.

[7] T. Kanstad, "Contribution to benchmark test no. 1, 2 and 3", Creep and shrinkage of concrete, RILEM TC 114 subcommittee 4, 901-911, 1993.

[8] S. Kim, S. Lee, "The behavior of reinforced concrete columns subjected to axial force and biaxial bending", Eng. Struct. 23, 1518-1528, 2000.

[9] Matlab, The language of technical computing, The Mathworks, Inc., 1999.

[10] B. Vratanar, M. Saje, "A consistent equilibrium in a cross-section of an elasticplastic beam", Int. J. Solids Structures 36, 311-337, 1999.

[11] D. Zupan, M. Saje, "A new finite element formulation of three-dimensional beam theory based on interpolation of curvature", Computer Modeling in Engineering and Sciences 4, 301-318, 2003.

[12] D. Zupan, M. Saje, "Finite-element formulation of geometrically exact threedimensional beam theories based on interpolation of strain measures", Comput. Methods Appl. Mech. Engrg. 192, 5209-5248, 2003. 
[13] D. Zupan, M. Saje, "The three-dimensional beam theory: finite element formulation based on curvature", Comput. Struct. 81, 1875-1888, 2003. 